\title{
Modelling and Simulation of Automated Underwater Acoustic Communication Transmission Recovery for Oceanic Robotics Operations
}

\author{
C. Chen $^{\text {a }}$, A. Anvar ${ }^{\mathrm{a}}$ and T. Lu ${ }^{\mathrm{a}}$ \\ ${ }^{\mathrm{a}}$ The University of Adelaide, School of Mechanical Engineering, Adelaide, South Australia, 5005 \\ Email: chen.chen@adelaide.edu.au
}

\begin{abstract}
In the area of Maritime Robotics a successful operational scenario would highly depend on clear acoustic communication-interaction of submersible Intelligent-Robot(s) and human-operator. The aim of this paper is to exhibit an analytical model to automatically recover underwater acoustic communication, digital-data transmission lost of the autonomous underwater vehicle (AUV) with human operator(s) in the loop. In this case an existing error-control code model based on Reed Solomon code is reviewed and tested. Then, the communication system of the AUV which requires a methodology of data loss recovery is discussed. Furthermore, the integration of a acoustic communication system and Reed Solomon code is discussed. This is followed by a simulation showing the robustness of an integrated data-communication system. The simulation results illustrate that the proposed communication scheme can not only withstand unforeseeable noise, but also allows for the transmission of digital data at high speed.
\end{abstract}

Keywords: AUV, underwater communication, Reed Solomon code, acoustic communication, Automation 


\section{INTRODUCTION}

As approximately $71 \%$ of the Earth's surface is covered by ocean, underwater communication plays an essential role in human life. Reliable underwater communication is always difficult because of the dynamic underwater ocean environment. The acoustics signal would be affected by not only ambient and man made noise but also path loss such as attenuation, geometric spreading and multi-path propagation as pointed out by Akyildiz et al. (2005). To achieve more reliable communication, an error control codes system could be integrated with an underwater communication system. Examples of commonly used error control codes include Hamming codes, Golay codes and Reed-Solomon codes. This branch of science was first introduced by Shannon (2001). The fundamental ideal introduced in this paper is to add some redundancies to the original information. Those redundancies can then be used to detect the error and even to correct the errors. The more redundancies added to the system, the more reliable the communication system is. On the other hand, the redundancies would slow the underwater communication system, which has smaller bandwidth compared with terrestrial communication. This results a tradeoff between usable data rate (the ratio of the usable data to the encoded data) and the reliability (the ability to detect and correct errors) of the communication system.

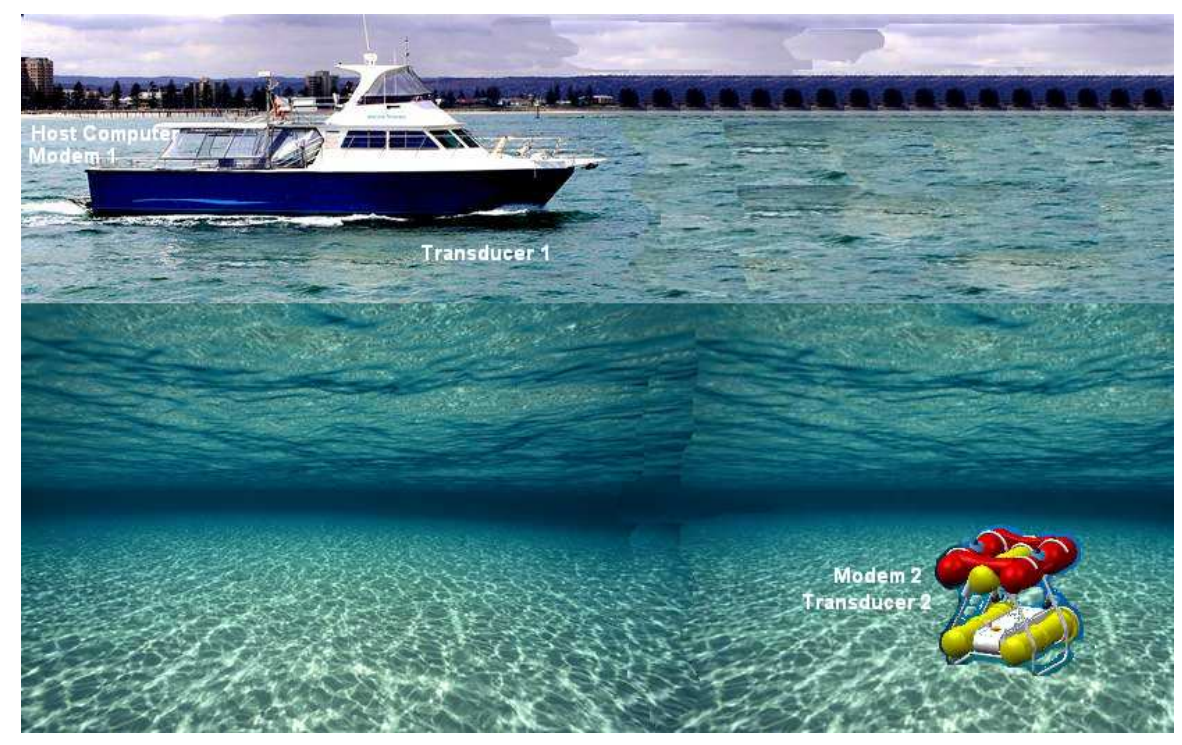

Figure 1: Underwater Communication System

A coding scheme designed for underwater acoustic communication with low bit rate and low power consumption is proposed by Green and Rice (1997). This coding scheme was shown to be robust with bit rate of 6.3 bits per second. However, with recent technology, the bit rate of acoustic sensors could be much higher. Later on, in the paper published by Cox et al. (2008), an underwater optical communication scheme with Reed-Solomon Code with codeword length of 255 and message length of 129 was demonstrated. Optical communication has a faster bit rate but suffers from low communication range due to scattering, as highlighted by Akyildiz et al. (2005). The proposed $(255,129)$ Reed Solomon error control code is shown to be robust for underwater optical communication. Although the type of communication sensor is different, the success of $(255,129)$ Reed Solomon code affords us experience that merits attention. However, acoustic is different from optical. As highlighted by Preisig (2007), the propagation characteristics of acoustics underwater highly depends on local environmental factors such as distance from the ocean surface and depth to the sea-bed as well as salinity and temperature in the water.

As a result, an error control code system that is designed for one particular depth may not work well for a different depth. For example a coding system with high redundancies is required for shallow water transmission since the acoustic signal could be reflected by the ocean surface randomly. In fact in a series of experiments by Bevan et al. (2010) showed that up to $20 \%$ of the signal could be lost due to the reflection from the ocean surface for the particular set of equipment they used.

In the work presented here the acoustic transducer (DSPComm Aquacomm), shown in Figure 2, was 


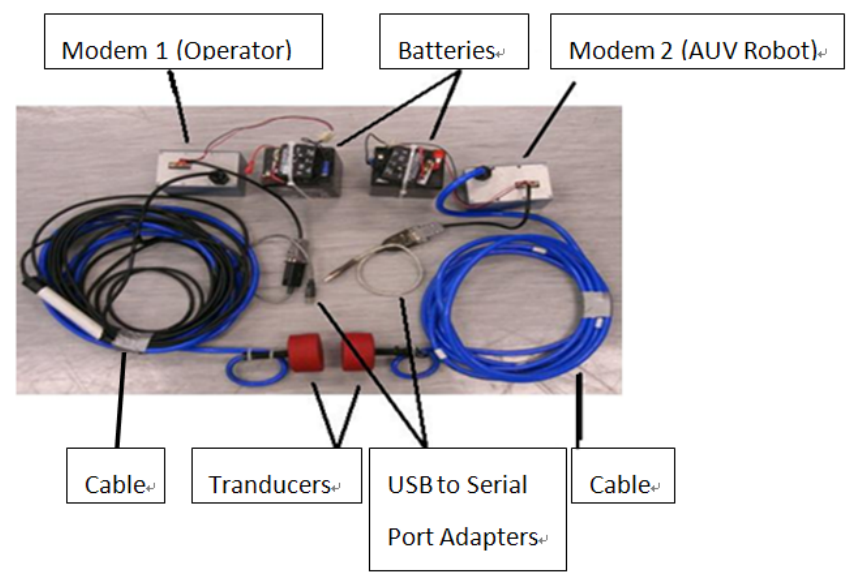

Figure 2: DSPComm Aquacomm Underwater Communication Equipments

used. This system has a better performance in deep ocean and a better transmission rate is achieved by reducing the redundancies built into the coding system as the AUV goes deeper into the ocean. An automated system based on a Reed Solomon Code is proposed to optimize the system's transmission rate and reliability.

In this paper, an automated acoustic communication system based on Reed Solomon Codes is proposed to optimize the system's transmission rate and reliability. The remainder of this paper is organized as follows. Section 2 introduces the background relevant to the proposed scheme. The details of the proposed scheme are described in Section 3. In Section 4, the simulation results are presented to demonstrate the effectiveness of the proposed scheme. Summary, conclusions and future work are finally drawn in Section 5.

\section{BACKGROUND}

\subsection{Cyclic Code}

The codeword of a cyclic code is often regarded as the coefficients of a polynomial:

$p(x)=\sum_{i=0}^{n-1} C_{i} x^{i}$

Where $\left(c_{0}, c_{1}, \cdots, c_{n-1}\right)$ are elements in a finite field. In this paper, only the finite field $G F\left(2^{8}\right)$ is used because of the popularity of 8-bit computer systems. The received codeword $p(x)$ is a correct codeword if and only if it is a multiple of the generator matrix $g(x)$. Let $m(x)$ be the polynomial whose coefficients are the message to be sent. Then, $p(x)=m(x) g(x)$ (over the polynomial ring $\mathbf{F}_{2^{8}}[x] /\left(x^{n}-1\right)$ ). Obviously, the degree of $p(x)$ (codeword length $n$ ) is equal to the sum of degree of $m(x)$ (message length $k$ ) and the degree of $g(x)$ (number of redundancies $\delta=n-k$ ). The Code Rate, is defined as the ratio of message length to codeword length:

$\mathcal{R}=\frac{k}{n}$

, is a parameter which indicates the rate of communication compare with the non-coded system. A cyclic code with codeword length $n$ and message length $k$ is usually referred as $(n, k)$ cyclic code.

\subsection{Reed-Solomon Codes in $G F\left(2^{8}\right)$}

Reed-Solomon is a powerful cyclic code which has been successfully applied to a variety of applications such as HDTV, CDs/DVDs, Blu-Ray Discs and DSL from Wicker and Bhargava (1999). Reed-Solomon 
code is a cyclic code generated by polynomial of the form:

$g(x)=\left(x-\alpha^{a+1}\right)\left(x-\alpha^{a+1}\right) \cdots\left(x-\alpha^{a+\delta-1}\right)$

Where $\alpha$ is a primitive element of $\mathbf{F}_{2^{8}}$ and $\delta$ is the number of redundancies.

\subsection{Hamming Distance}

The commonly used quantity to indicate reliability of an error-control code is Hamming Distance $d$ which is first defined by Hamming (1950). Hamming Distance $d$ between two messages which have the same length is defined as the number of positions at which the corresponding symbols are different. For example, Hamming Distance between the message "dive" and "move" is 2 because "di" and "mo" are different symbols. It is also proved that a coding system with Hamming Distance $d$ can detect up to $d-1$ errors in a codeword. Also, the number of errors a coding system with Hamming Distance $d$ can correct is is presented as:

$c=\left\lfloor\frac{d-1}{2}\right\rfloor$

\section{Proposed Scheme}

\subsection{Comparison between Reed-Solomon Codes and Other Codes}

As stated by Richardson and Urbanke (2008), codes are classified into convolutional code and block code. Convolutional code is usually decoded by Viterbi decoder in exponential time, as pointed out by Feldman et al. (2002), whereas a block code can be decoded in polynomial time. Thus, convolutional code would give the communication system more computational load with high complexity system. Typical block codes include Hamming codes, Golay codes and Cyclic codes. Hamming code is not applicable for the purpose of underwater communication since it can only correct a single error in a message. The underwater noise level could vary dramatically, thus the redundancy of coding system is expected to adjust automatically. Golay code is not suitable in this case because the code rate is fixed. Cyclic code is suitable for the purpose because of its multiple error correction ability and its flexibility with message length and codeword length. Reed-Solomon codes, which is the most widely used cyclic code, can be implemented into underwater communication system, see Cox et al. (2008).

\subsection{Speed and Reliability}

From the definition of Reed Solomon Codes, it should be highlighted that there is a trade-off which can be described as follows: for a given class of error control code and the length of message $k$, the codeword length $n$ has to be greater than message length $k$. To increase reliability of the communication system, more redundancies $\delta$ are required. It means a larger value for $n$ is required. As codeword length $n$ increases, the code rate $\mathcal{R}$ decreases. This will slow the communication system in two ways:

1. Smaller $\mathcal{R}$ leads to heavier computational load. Hence it become slower to encode and decode the message.

2. The size of encoded message become larger as $\mathcal{R}$ is reduced. Hence the time taken to transmit message is longer.

A desired coding system will maximise the speed while Hamming Distance $d$ is large enough to ensure the success of the data transmission. For a Reed Solomon Code system, the Hamming Distance $d$ can be calculated by the formula shown by Clark and Cain (1982):

$d=n-k+1$

By equation 5, by increasing codeword length $n$, Hamming Distance $d$ will increase. By combining equation equation 5 with 4, the number of errors Reed Solomon Code can correct is also shown as:

$c=\left\lfloor\frac{n-k}{2}\right\rfloor$ 


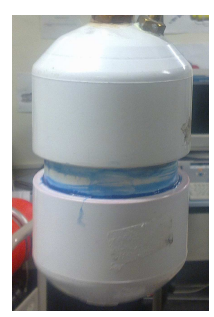

(a) Pressure Sensor

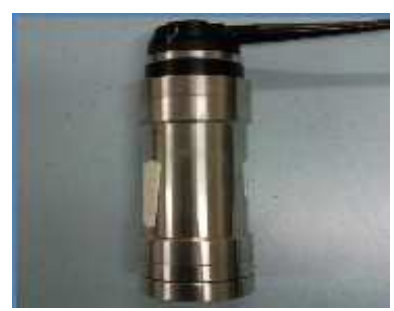

(b) Altimeter

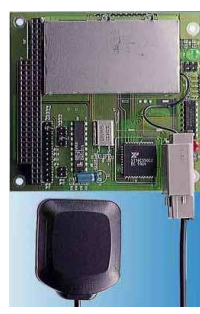

(c) GPS

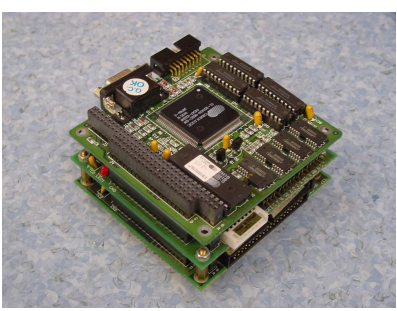

(d) Imbedded PC104

Figure 3: Sensors in AUV

According to equation 5, by fixing the codeword length $n$, there is a one to one correspondence between the number of error a Reed Solomon Code can correct $c$ and the message length $k$. Ideally, when the depth from water surface $h$ is large, a $k$ needs to be close to $n$ to minimise the code rate $\mathcal{R}$. When the depth from water surface $h$ is small, $k$ needs to be small to maximise the Hamming Distance. To attain the distance from surface and depth to the sea-bed, a pressure sensor (Figure 3a) and an altimeter sensor (Figure $3 b$ ) are in action and would report the real-time passive and active signals to the AUV Robot Controller.

\subsection{Simulation}

This research focused on the problem of acoustic reflection in shallow water, the distance of the AUV from the ocean surface will dominant the data transmission performance rather than the distance between two acoustic modems. Accoding to Bevan et al. (2010), the variation of distance between two acoustic modems from 10 metres to 40 metres will not affect the acoustic transmission. Within this range of distance, the proportion of successful communication can be modelled as a function of depth. The steps of the simulation is described as follows. First, message length $k$ is computed according to the depth $h$ measured by data from pressure sensor or altimeter. Three different cases to compute $k$ are:

1. When the AUV is operating in shallow water, the ocean surface reflection will dominant the acoustic signal transmission performance. The experimental result recorded by Bevan et al. (2010) showed that, within 40 metres range, during testing in ocean when the water is shallower than 3 metres ( $h \leq 3$ metres) and, up to $20 \%$ of the data was lost. $(255,129)$ Reed Solomon code suggested by Cox et al. (2008) is used due to its ability to correct numerous bit errors.

2. When the AUV is navigating within deeper water ( $h \geq 10$ metres), the acoustic system should perform well since the acoustic transducer is designed for deep water. In this case, $(255,223)$ Reed Solomon code can be used and that is its due to its capability in which can correcting 16 errors in a single message.

3. Interpolation is used between previous cases. When the depth of AUV is between 3 metres and 10 metres, the number of errors in transmitted codeword is estimated according to the depth measured by data from pressure/altimeter sensors by equation 7. According to the number of errors, the system will compute Hamming Distance $d$ required for reliable communication and hence the messaged length $k$.

Now, the underwater communication message can be encoded with $(255, k)$ Reed-Solomon code and be sent. Before sending the message, the other terminal need to be informed the message length $k$ in order to decode. To ensure $k$ can be received by the other terminal, $k$ will be decoded by $(255,129)$. The other terminal will decode the message when message is received. After each simulation, the elapsed time for encoding and decoding are saved respectively, and a simulation with same procedure but fixed $(255,129)$ Reed-Solomon will be performed to compare with proposed scheme.

Encoding. Encoding has several steps as follows:

1. Compute the the message length $k$ according to the depth $h$ measured by pressure sensor by the 
following rule:

$$
k= \begin{cases}129 & h \leq 3 \\ 2\left\lfloor 64.5+\frac{(h-3)}{7} 47\right\rfloor+1 & 3<h<10 \\ 223 & h \geq 10\end{cases}
$$

2. Send the value $k$ with $(255,129)$ Reed-Solomon code.

3. Encode the message with the $(255, k)$ Reed-Solomon code.

4. Send by the acoustic system. The system consists of a high-level of $\mathrm{C}++$ program and two acoustic modems.

Decoding. The steps of Decoding is as follows:

1. Decoded the codeword to the message with $(255, k)$ Reed-Solomon codes using the algorithm proposed by Clark and Cain (1982).

2. If the message is unreadable, ask for retransmission with $(255,129)$ Reed-Solomon code.

\section{Simulation Result}

\subsection{Code Rate}

The purpose of automated $(255, k)$ Reed-Solomon codes is to increase the code rate in deep water. The reason is the DSPComm Aquacomm acoustic transducer performed much reliable in deeper part of shallow water. As shown in Figure 4, the code rate rise from 0.51 to 0.88 as the the AUV dives deeper. This leads to 32 less information to be sent and will improvement the speed of communication system considerably.

\subsection{Computational Improvement}

The improvement of the proposed system is shown in Figure 5 by comparing with a fixed $(255,129)$ Reed-Solomon coding system. The horizontal axis represents the distance of AUV from ocean surface in metres. The quantity in vertical axis represents the percentage speed improvement for encoding and decoding. Namely, it represents the elapsed time for encoding or decoding with an automated $(255, k)$ Reed-Solomon over the elapsed time for encoding or decoding with fixed $(255,129)$ Reed Solomon code for a same message. The figure shows that there is an improvement in encoding computational efficiency and a larger improvement in decoding computational efficiency. The simulation suggest the coding system could be more than 10 times faster than the fixed $(255,129)$ Reed Solomon code.

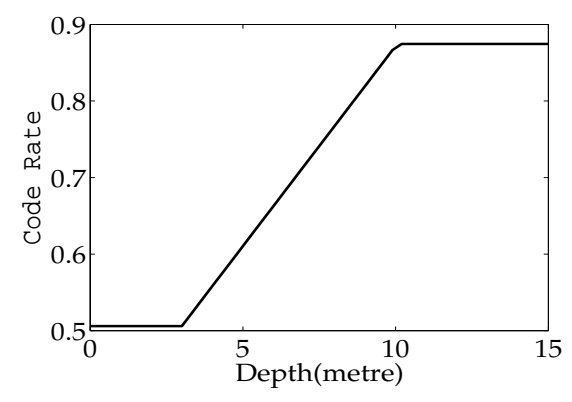

Figure 4: Code Rate vs Depth

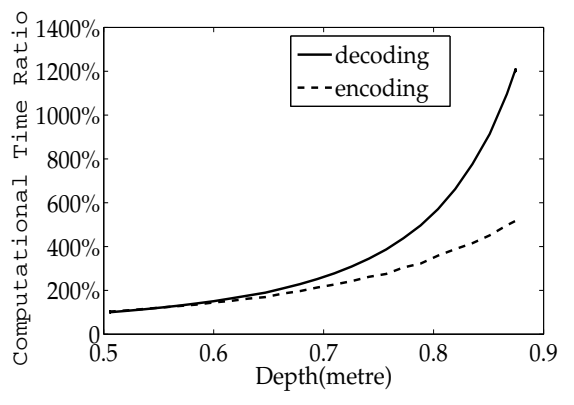

Figure 5: Computational Improvement vs Depth 


\section{CONClusion ANd Future Work}

Error control code is widely applied in underwater communication system, as pointed out by Green and Rice (1997). In this paper, an acoustic communication system using automated Reed-Solomon Code is proposed. In the initial stage, the proposed scheme identifies a suitable message length $k$ according to the distance from water surface $h$. For the next stage, the message is encoded with automated $(255, k)$ code and transmitted to the other terminal. In the process of decoding, the algorithm proposed by Clark and Cain (1982) is applied. In the investigation, the simulation results show that the proposed scheme not only can successfully transmit message under noisy underwater environment, but also can transmit in a fast rate. In addition, the proposed scheme is robust as it reduces the require of computational load. The future work includes with the AUV communication system trials and tests within deeper part of the shallow water ocean.Also, a optical communication sensors could be considered and tested as another possible option of the AUV's shallow water communication system.

\section{ACKNOWLEDgment}

The authors would like to thank the contribution of honour's team of 998 in 2010 of the School of Mechanical Engineering, the University of Adelaide and also thank to the staff at the electronic workshop of the School of Mechanical Engineering.

\section{REFERENCES}

Akyildiz, I. F., D. Pompili, and T. Melodia (2005). Underwater acoustic sensor networks: research challenges. Ad Hoc Networks 3(3), 257 - 279.

Bevan, J., D. L. Zhao, X. Mu, and N. H. Yahaya (2010, October). Intelligent robot long-distance hybrid secure communication control. Honour Thesis.

Clark, J. G. C. and J. B. Cain (1982, August). Error-correction coding for digital communications (2. print. ed.), Volume Second Printing. New York: Plenum Press.

Cox, W., J. Simpson, C. Domizioli, J. Muth, and B. Hughes (2008, sept.). An underwater optical communication system implementing reed-solomon channel coding. In OCEANS 2008, pp. 1 -6.

Feldman, J., I. Abou-faycal, and M. Frigo (2002). A fast maximum-likelihood decoder for convolutional codes. In Vehicular Technology Conference.

Green, M. and J. Rice (1997, October). Error correction coding for communication in adverse underwater channels. In OCEANS '97. MTS/IEEE Conference Proceedings, Volume 2, pp. 854 -861 vol.2.

Hamming, R. W. (1950). Error detecting and error correcting codes. Bell System Technical Journal 29(2), $147-160$.

Preisig, J. (2007, October). Acoustic propagation considerations for underwater acoustic communications network development. SIGMOBILE Mob. Comput. Commun. Rev. 11, 2-10.

Richardson, T. and R. Urbanke (2008, March). Modern Coding Theory. Cambridge University Press.

Shannon, C. E. (2001, January). A mathematical theory of communication. SIGMOBILE Mob. Comput. Commun. Rev. 5, 3-55.

Wicker, S. and V. Bhargava (1999). Reed-Solomon Codes and Their Applications. John Wiley \& Sons. 